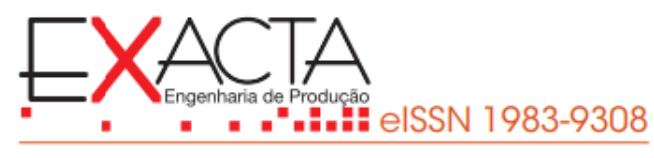

\title{
ALTERNATIVAS DE MELHORIA DA QUALIDADE NOS PROCESSOS DE UM RESTAURANTE SOB A ÓTICA DAS PRÁTICAS SUSTENTÁVEIS
}

\section{ALTERNATIVES OF QUALITY IMPROVEMENT IN THE PROCESSES OF A RESTAURANT UNDER SUSTAINABLE PRACTICES}

Recebido em: 06 jun. 2019

Aprovado em: 13 fev. 2020

Versão do autor aceita publicada online: 13 fev. 2020

Publicado online: 18 maio 2021

Como citar esse artigo - American Psychological Association (APA):

Silva, D. J. C., Johann, D. A., Nunes, A. de F. P., Serpa, N. P., \& Pozzer, R. H. P. (2021, out./dez.). Alternativas de melhoria da qualidade nos processos de um restaurante sob a ótica das práticas sustentáveis. Exacta. 19(4), 764-784. https://doi.org/10.5585/exactaep.2021.14166.

Submeta seu artigo para este periódico $\bigoplus$

Dados Crossmark 


\title{
ALTERNATIVAS DE MELHORIA DA QUALIDADE NOS PROCESSOS DE UM RESTAURANTE SOB A ÓTICA DAS PRÁTICAS SUSTENTÁVEIS
}

\author{
ALTERNATIVES OF QUALITY IMPROVEMENT IN THE PROCESSES OF A RESTAURANT \\ UNDER SUSTAINABLE PRACTICES
}

(iD) Deoclécio Junior Cardoso da Silva
(iD Denise Adriana Johann ${ }^{2}$
(i) Andrieli de Fátima Paz Nunes ${ }^{3}$
(iD Natalia Pedroso Serpa ${ }^{4}$
(iD Ranice Höehr Pedrazzi Pozzer ${ }^{5}$

${ }^{1}$ Mestre em Engenharia de Produção. Universidade Federal de Santa Maria - UFSM. Santa Maria, Rio Grande do Sul - Brasil. deocleciojunior2009@gmail.com

${ }^{2}$ Mestra em Administração. Universidade Federal de Santa Maria - UFSM. Santa Maria, Rio Grande do Sul - Brasil. johanndenisee@gmail.com

${ }^{3}$ Mestra em Administração. Universidade Federal de Santa Maria - UFSM. Santa Maria, Rio Grande do Sul - Brasil. andriieli.nunes@gmail.com

${ }^{4}$ Mestra em Engenharia de Produção. Universidade Federal de Santa Maria - UFSM. Santa Maria, Rio Grande do Sul - Brasil. nataliapedrososerpa@gmail.com

${ }^{5}$ Doutora em Administração Universidade Federal de Santa Maria - UFSM. Santa Maria, Rio Grande do Sul - Brasil. ranice@gmail.com

Recebido em: 06 jun. 2019

Aprovado em: 13 fev. 2020
Resumo: Diante aos aspectos inerentes que permeiam a temática da sustentabilidade, bem como a importância desta na sociedade, torna-se relevante que estudos sejam realizados com a finalidade de auxiliar as organizações a estarem alinhadas a esse viés. Dessa forma objetivo do presente estudo é avaliar as alternativas de melhoria na qualidade no processo com ênfase nas práticas sustentáveis em um restaurante situado na região noroeste do Rio Grande do Sul. Através de uma pesquisa exploratória, descritiva, de abordagem qualitativa e quantitativa, este estudo de caso avaliou um restaurante, elencando alternativas de melhoria da qualidade, alinhadas a práticas sustentáveis, priorizando através do método de Análise hierárquica de processos (AHP) aquela que na visão do gestor é mais relevante. Dessa forma, os resultados demonstraram que alternativa voltada a treinamento do pessoal é a mais priorizada, visto sua influência direta nos processos, o que pode auxiliar na redução dos desperdícios no restaurante.

Palavras-chave: Sustentabilidade em restaurantes. Análise hierárquica de processos. Práticas sustentáveis.

Abstract: In view of the inherent aspects that permeate the sustainability theme, as well as the importance of sustainability in society, it is relevant that studies are carried out with the purpose of helping organizations to be aligned with this bias. Thus, the objective of the present study is to evaluate the alternatives for improvement in quality in the process with emphasis on sustainable practices in a restaurant located in the northwest region of Rio Grande do Sul. Through an exploratory, descriptive, qualitative and quantitative approach, this study of cases evaluated a restaurant, listing quality improvement alternatives, aligned to sustainable practices, prioritizing through the hierarchical Process Analysis (AHP) method that the manager's view is more relevant. Thus, the results showed that an alternative to personnel training is the most prioritized, since its direct influence on the processes, which can help reduce the waste in the restaurant.

Keywords: Sustainability in restaurants. Analytic hierarchy process. Sustainable practices. 


\section{Introdução}

O desenvolvimento sustentável está relacionado ao uso dos recursos, de forma a suprir necessidades individuais, sem impactar significativamente o meio ambiente e assegurando disponibilidade de recursos no futuro, o que, segundo Ulutaş et al (2019) só será possível se as questões sociais, econômicas e ambientais forem consideradas. Os autores destacam que numerosos estudos demonstram que a preocupação com o ambiente, aliada a objetivos relacionados ao desenvolvimento social e econômico contribuem não apenas para os governos, mas para as organizações privadas.

Em relação ao uso dos recursos, o desenvolvimento tecnológico tem proporcionado a implementação de melhorias nos processos produtivos. Entretanto, se por um lado, é possível produzir mais e com mais qualidade (Porter, 1996), com maior expectativa de vida e com menores distâncias com a internet (Aguiar, 2002), por outro, apesar dessas melhorias, os recursos necessários ultrapassaram a capacidade do planeta de renová-los. Madruga (2009) destaca que "o desafio de superar a conduta individualista, presente em uma expressiva parcela da população mundial, que se lança ao consumo e ao uso abusivo de recursos naturais, acreditando que suas fontes são inesgotáveis" (Madruga, 2009, p.17) é uma das principais questões relacionadas à sustentabilidade. Em atenção a esta realidade, diversos segmentos da sociedade têm buscado novos padrões de produção e consumo.

Nesse sentido, em 2015, a Organização das Nações Unidas (ONU), em um esforço para fomentar o desenvolvimento de práticas sustentáveis nas mais diversas áreas, definiu os Objetivos de Desenvolvimento Sustentável globais, a Agenda 2030. São 17 objetivos que buscam redução da pobreza a partir do estabelecimento de 169 metas nas dimensões econômica, social e ambiental. Entre os objetivos da ONU, o ODS-12 interessa particularmente ao presente estudo, pois relaciona-se a busca por padrões de produção e consumo sustentáveis, com foco na gestão sustentável e uso eficiente de recursos naturais, redução do desperdício de alimentos nos níveis de varejo e do consumidor e nas cadeias de produção e abastecimento (ONU, 2015). O presente estudo está alinhado com a Agenda 2030, pois objetiva analisar as alternativas de melhoria na qualidade nos processos, com ênfase nas práticas sustentáveis em um restaurante situado na região noroeste do Rio Grande do Sul.

Para analisar as práticas sustentáveis no restaurante objeto deste estudo, esta pesquisa utiliza como técnica o modelo multicritério Análise Hierárquica de Processos (AHP). A ferramenta AHP (Lee, 2010; Ho, Dey \& Lockström, 2011; Peterková \& Franek, 2018; Ergün \& Bülbül, 2019) tem sido utilizada em problemas multicritério complexos. Para este estudo, foram estabelecidos quatro critérios para análise das práticas sustentáveis: qualidade, custos, tempo e recursos. O critério qualidade está voltado aos processos, permitindo melhorias nas três dimensões da sustentabilidade (Kin \& Shim, 2019). O critério custos volta-se à dimensão econômica, com busca por redução de custos e controle na produção e na geração de resíduos (Zhu et al., 2019). O critério tempo também influencia a dimensão 
econômica da sustentabilidade, estando diretamente relacionado ao volume de produção e variação nos custos (Zhang et al., 2019). O último critério envolve a utilização racional dos recursos, que estaria voltada à diminuição dos impactos ambientais e sociais (Pakdil, Toktas \& Leonard, 2018). A partir desses critérios, foram definidas alternativas para melhoria da qualidade nos processos do restaurante, visando estabelecimento de práticas sustentáveis adotadas pela empresa. As alternativas foram treinamento de pessoal, Programa 5S, reorganização de layout, gerenciamento e armazenagem de matéria-prima, estoque e padronização do tempo.

O presente artigo está estruturado em cinco seções: a primeira é introdutória; a segunda apresenta o referencial teórico e discorre sobre conceitos referentes a qualidade nos processos e sustentabilidade; a terceira é dedicada à apresentação da metodologia de pesquisa; a quarta apresenta os resultados e discussões e a quinta seção apresenta considerações finais e possibilidades para futuras pesquisas.

\section{Referencial teórico}

\subsection{Sustentabilidade e ações sustentáveis aplicadas em restaurantes}

A sustentabilidade, tema expressivo em todo o mundo, vem ganhando força desde os anos 1970 através da conscientização dos consumidores com relação do desenvolvimento e produção de produtos e serviços ecologicamente corretos (Brandalise et al. 2009; Trudel, 2019; Garcia et al., 2019; Zhu \& Yu, 2019).

No que tange aos restaurantes, cada vez mais tem se entendido e estudado formas para que haja redução de desperdícios, bem como para que os resíduos gerados por eles sejam reaproveitados e atitudes sejam modificadas (Raab, Baloglu \& Chen, 2018; Alegre \& Berbegal-Mirabent, 2018; Koo, 2018; Michalec et al., 2018; Vinck, Scheelen \& Du Bois, 2019; Salzberg, Gough \& Suen, 2019).

Nesse sentido, o estudo realizado por Hashem et al. (2019) trata da produção de biocombustíveis alternativos a partir de bioresíduos ricos em amidos gerados por restaurantes. Esses resíduos, que em restaurantes de países em desenvolvimento são produzidos em grandes quantidades, representam um substrato sustentável para a produção de etanol. Os autores estudaram a viabilidade da conversão dos resíduos amiláceos em biocombustível (bioetanol), recomendando a aplicação dessa tecnologia em escala comercial em todo o mundo para o beneficiamento de bioresíduos. Sob a ótica da sustentabilidade, as descobertas do estudo oferecem uma solução para a reciclagem de resíduos, produzindo biocombustível ecologicamente correto.

Teng \& Wu (2019), buscando identificar o que determina o desenvolvimento sustentável dos restaurantes verdes, verificaram que valores utilitários afetam de forma significativa e positiva a preferência e intenção dos consumidores por restaurante verdes. 
Tommasetti et al. (2018), a partir de estudo baseado na prátic Estendida do Comportamento Planejado, buscaram identificar as variáveis capazes de orientar os consumidores para a escolha de restaurantes sustentáveis. Os resultados da análise forneceram evidências empíricas para as hipóteses formuladas sugerindo que a atitude, a norma subjetiva, o controle comportamental percebido, a utilidade percebida e a curiosidade afetaram a intenção comportamental dos consumidores em preferir restaurantes sustentáveis, e que a intenção comportamental afetou seu comportamento real.

Bharucha (2018) desenvolveu seu estudo voltado ao desperdício de milhões de toneladas de alimentos na Índia, com objetivo em concentrar-se em uma fonte importante para a qual a atual proporção maciça de desperdício pode ser atribuída: restaurantes. O autor argumenta que os restaurantes precisam monitorar e minimizar o desperdício de alimentos, para o bem da comunidade e do planeta, apesar do resultado do seu estudo demonstrar uma fraca associação entre atitudes e comportamentos de prevenção de resíduos com ganhos enormes entre comportamentos e valores pró-ambientais.

A partir dos estudos acerca da importância da sustentabilidade em restaurantes, verificou-se que as empresas inseridas no atual cenário competitivo buscam entender como alcançar o sucesso, sendo sustentáveis e ainda assim competitivas. Em diversos estudos os autores descrevem a sustentabilidade como uma das chaves para conquistar as quotas de mercado ou manter-se nele. Para tanto, torna-se relevante compreender a dinâmica entre consumo, produção e sustentabilidade.

\section{Metodologia}

Nos diversos âmbitos de estudos uma pesquisa científica nasce do senso comum, uma vez que o mesmo emerge da necessidade de resolver adversidades imediatas por meio de práticas não planejadas, uma vez que o senso comum se trata daquilo que não é ciência, afirmando-se que é a partir dele, que surgem estudos acadêmicos que auxiliam para atender as necessidades e demanda da sociedade (Cauchick Miguel, 2012; Martins Mello \& Turrioni, 2014). Nesse sentido, os procedimentos metodológicos são de suma importância na realização de uma pesquisa, especificando técnicas de coleta e estratégias analíticas que serão aplicadas. Outro determinante que ilustra a importância que os procedimentos metodológicos desempenham é o fato de que, a partir deles, se chegará ao objetivo geral da pesquisa, guiando todo o andamento do estudo (Marconi \& Lakatos, 2010; Demo, 2013).,

Em virtude disso, o método adotado na presente pesquisa é apresentado no apresentado no Figura 1. 
Figura 1

Processo metodológico da pesquisa

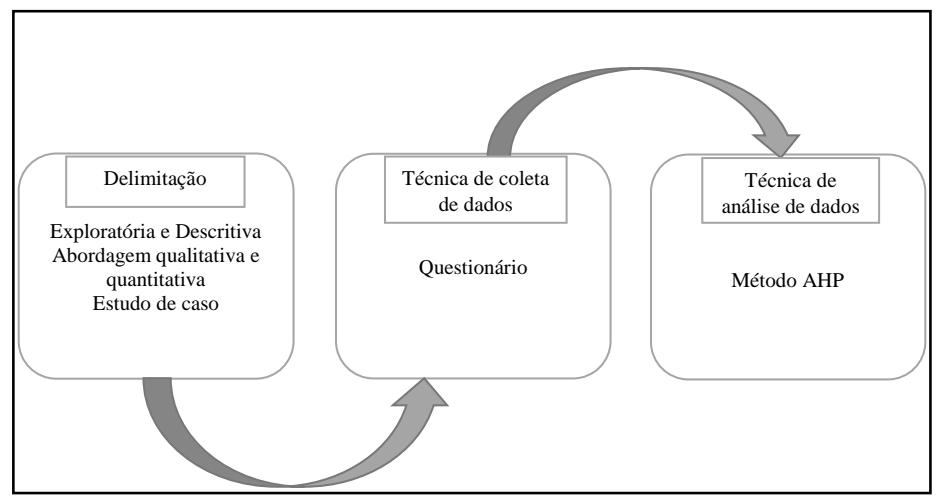

Fonte: Elaborado pelos autores.

A presente pesquisa foi realizada em restaurante sendo delimitado como um estudo exploratório, pois se buscou ter maior familiaridade com o assunto, onde por meio de observações, verificou-se como é a percepção do gestor quanto a qualidade nos processos, priorizando alternativas sob a ótica da sustentabilidade (Ruiz, 2011). Em concordância, a presente pesquisa enquadrou-se na abordagem descritiva (Collies \& Hussey, 2005; Gil, 2010; Marconi \& Lakatos, 2010; Appolinário, 2011).

Nesta pesquisa, utilizou-se uma abordagem combinada quanto a natureza dos dados, definindo-se este estudo como qualitativo e quantitativo. Tal posicionamento se justifica pela consideração que as vantagens de uma abordagem amenizam as desvantagens da outra (Cauchick Miguel, 2012). Para atender aos interesses desse artigo, definiu-se que a pesquisa se trata de um estudo de caso. (Cauchick Miguel, 2012; Martins, Mello \& Turrioni, 2014), uma vez que a pesquisa para avaliação das alternativas de melhoria na qualidade nos processos, com ênfase nas práticas sustentáveis em um restaurante situado na região noroeste do Rio Grande do Sul, justifica essa classificação.

\subsection{Coleta e análise de dados}

No que tange a coleta e análise de dados, afirma-se que se deu em dois momentos distintos.

Visando alcançar o objetivo proposto a pesquisa foi separada em três etapas, desde a definição da empresa a ser analisada até a ponderação dos critérios e ranqueamento das alternativas. Nesse sentido, a metodologia proposta que contempla a coleta e análise dos dados é ilustrada na Figura 2, sendo a mesma composta por 3 etapas. 
Figura 2

Metodologia proposta para coleta e análise dos dados da pesquisa

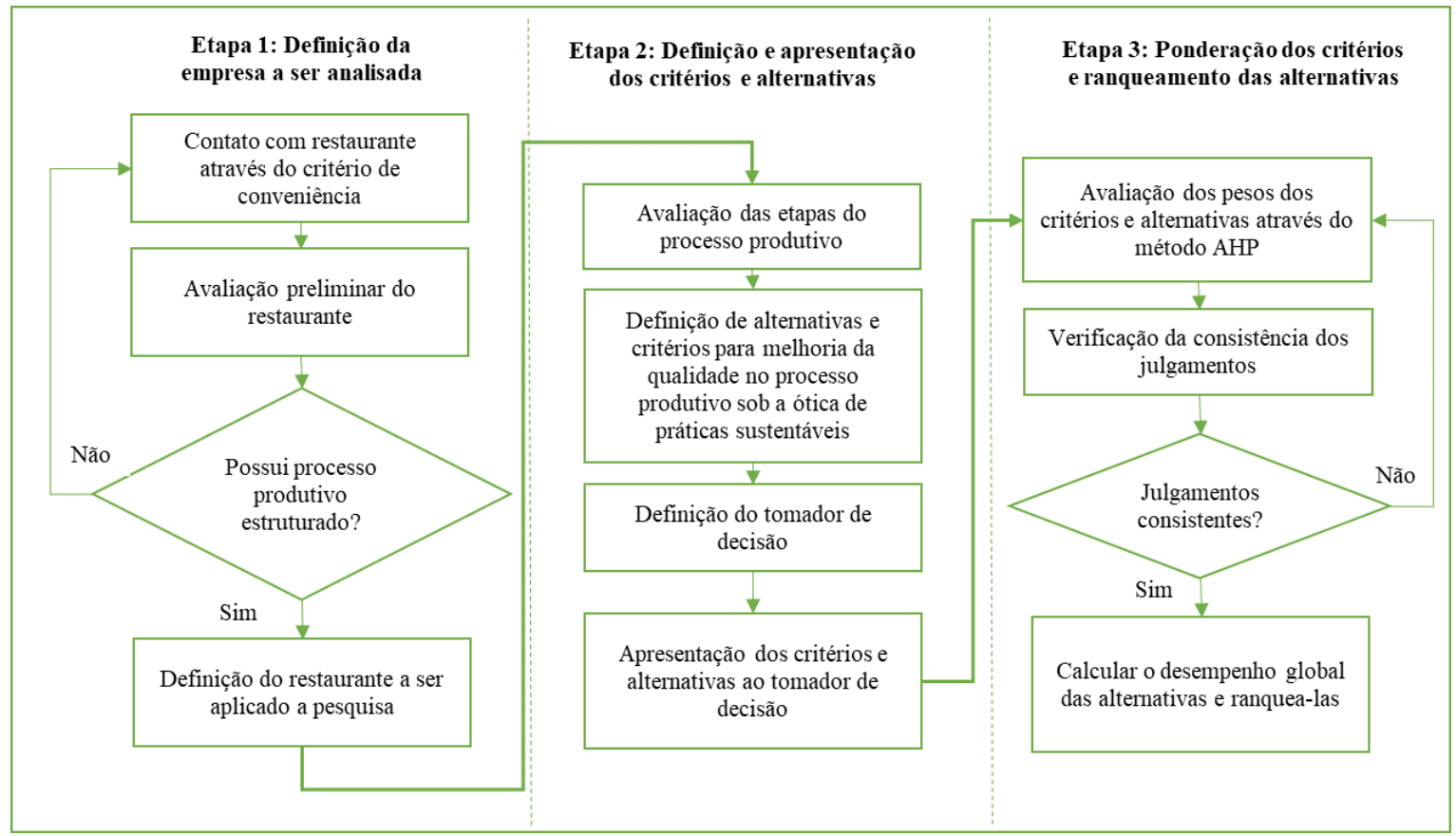

Fonte: Elaborado pelos autores.

Na etapa 1, foi necessário estabelecer contato com os restaurantes na região estudada, onde através do critério de conveniência os pesquisadores explicavam a pesquisa aos gestores e os convidavam a participar. Caso demonstrasse interesse pelo estudo, os pesquisadores realizavam uma análise preliminar do restaurante, visualizando principalmente o processo produtivo, visto que o objetivo da presente pesquisa está relacionado a esse contexto. No caso de haver um processo produtivo estruturado, ou seja, os produtos comercializados fossem produzidos no local o restaurante era escolhido para ser aplicada a pesquisa. Assim, escolheu-se o restaurante, visto que os demais ou não se enquadravam no critério apresentado, ou não tinham interesse de participar.

A etapa 2 teve como fase inicial uma avaliação mais profunda das etapas do processo produtivo, verificando os pontos a serem melhorados sob a ótica das práticas sustentáveis. Com essa análise, bem como com a utilização de material bibliográfico, foi possível definir os critérios e alternativas de melhoria da qualidade nos processos sob a ótica de práticas sustentáveis. Assim, o Quadro 1 apresenta os critérios definidos para serem analisados. 


\section{Quadro 1}

Critérios e suas contribuições para a sustentabilidade.

\section{Critérios}

\section{Contribuições}

A qualidade nos processos, bem como do produto devem estar atreladas diretamente à

Qualidade preocupação com o meio ambiente e a sustentabilidade. A partir disso, cabe ao gestor aplicar um sistema que racionalize a utilização de recursos e ao mesmo tempo garanta a satisfação de seus consumidores acerca do bem ou serviço prestado (Mastrapa Gutiérrez \& Sánchez Batista, 2017).

Custos Os processos de uma organização para serem considerados sustentáveis devem, anteriormente, passar por uma análise de custo-benefício. Deste modo é possível afirmar que um processo é viável e economicamente rentável para a empresa tornando-a competitiva frente ao mercado (Gigli, Landi \& Germani, 2019).

Tempo

O tempo de preparo e de produção influencia diretamente nos parâmetros econômicos da sustentabilidade. $E$ isso se reflete na quantidade de produtos e serviços ofertados gerando um menor lucro para a organização (Batista, Gouveia \& Marques, 2018).

Os recursos quando utilizados de forma racional contribuem para geração de melhorias nos três

Recursos pilares da sustentabilidade, reduzindo os custos de produção, os impactos ambientais originados pela criação de resíduos e ampliando motivação dos colaboradores da organização (Shen et al.,2019).

Fonte: Elaborado pelos autores.

Do mesmo modo, definiu-se alternativas sendo evidenciadas no Quadro 2, objetivando a melhoria da qualidade no processo do restaurante, levando em consideração alinha-lo as práticas sustentáveis. 


\section{Quadro 2}

\begin{tabular}{|c|c|}
\hline Alternativas & Contribuições \\
\hline $\begin{array}{l}\text { A - Treinamento } \\
\text { do Pessoal }\end{array}$ & $\begin{array}{l}\text { Treinar as competências dos colaboradores é um método primordial no } \\
\text { desenvolvimento de capital humano em uma empresa moderna. A formação pessoal } \\
\text { é um processo ao longo da vida e facilita o sucesso profissional e a carreira } \\
\text { contribuindo para o pilar social do tripé da sustentabilidade (Trelová \& Olšavský, } \\
\text { 2017). }\end{array}$ \\
\hline B - Programa 5S & $\begin{array}{l}\text { A metodologia } 5 S \text { modifica os comportamentos, bem como o ambiente de trabalho } \\
\text { de um departamento ou organização, colaborando para o trabalho em equipe, } \\
\text { melhorando a comunicação, aumentando a motivação do pessoal e atingindo } \\
\text { objetivos específicos. (Telles et al., 2013). }\end{array}$ \\
\hline $\begin{array}{l}\text { C - Reorganização } \\
\text { do Leiaute Físico }\end{array}$ & $\begin{array}{l}\text { Rearranjar o leiaute físico não só traz melhorias para a produtividade da empresa, } \\
\text { como contribui nos pilares econômico e social da sustentabilidade. No que tange à } \\
\text { dimensão econômica, uma boa organização física acarreta em uma maior eficiência } \\
\text { nos processos, consequentemente, reduzindo as perdas produtivas. Quanto ao pilar } \\
\text { social, o leiaute físico contribui para um maior bem-estar dos colaboradores, que } \\
\text { através de um espaço físico adequado, permite aos mesmos realizarem suas tarefas } \\
\text { com sucesso (Aghazadeh, 2005). }\end{array}$ \\
\hline $\begin{array}{l}\text { D - Gerenciamento } \\
\text { de matéria-prima }\end{array}$ & $\begin{array}{l}\text { Uma empresa que almeja se tornar competitiva e sustentável deve implementar } \\
\text { melhorias em sua forma de gerenciar a matéria-prima utilizada em sua produção. } \\
\text { Diante disso, a organização deverá buscar reduzir continuamente os impactos contra } \\
\text { o meio ambiente, adotando medidas de prevenção contra o desperdício de materiais } \\
\text { no âmbito produtivo (Florén et al., 2019). }\end{array}$ \\
\hline $\begin{array}{l}\text { E - Armazenagem } \\
\text { de matéria-prima }\end{array}$ & $\begin{array}{l}\text { Uma armazenagem inadequada da matéria-prima pode acarretar em perdas para a } \\
\text { organização. Esses desperdícios podem levar a altos custos produtivos que podem } \\
\text { tornar inviável um processo de uma organização. A armazenagem feita de forma } \\
\text { errônea contribui negativamente para o pilar econômico da sustentabilidade } \\
\text { (Shunmugasundaram \& Maneiah, 2018). }\end{array}$ \\
\hline F - Estoque & $\begin{array}{l}\text { Os benefícios que as práticas ambientais trazem para a organização são muitos, } \\
\text { entre eles, estão a redução de desperdícios dentro da organização. Onde, por } \\
\text { exemplo, o controle de estoques se faz necessário a fim de minimizar as perdas por } \\
\text { um acondicionamento inadequado dos produtos acabados. Assim, contribui } \\
\text { diretamente para os pilares econômico e ambiental da sustentabilidade (Nakuja \& } \\
\text { Kerr, 2018). }\end{array}$ \\
\hline $\begin{array}{l}\text { G - Padronização } \\
\text { do tempo }\end{array}$ & $\begin{array}{l}\text { Realizar a padronização do tempo é imprescindível para a sustentabilidade, pois ela } \\
\text { permite comedir a utilização ineficiente de energia elétrica, acerca da variação do } \\
\text { tempo de utilização das máquinas elétricas. A partir disso, é preciso um maior } \\
\text { controle sobre o tempo, ou seja, padroniza-los de modo a ampliar a produtividade } \\
\text { da organização (Raval, Kant \& Shankar 2018). }\end{array}$ \\
\hline
\end{tabular}

Fonte: Elaborado pelos autores.

Na etapa 3, tem-se a ponderação dos critérios e ranqueamento das alternativas. Visando realizar a análise dos dados obtidos, bem como priorizar os critérios mais relevantes utilizou-se como método de análise, o modelo multicritério "Análise hierárquica de processos" (AHP).

A ferramenta AHP (Lee, 2010; Ho, Dey \& Lockström, 2011; Peterková \& Franek, 2018; Ergün \& Bülbül, 2019) foi desenvolvida nos anos 70 pelo matemático Thomas L. Saaty e tem sido utilizada em problemas multicritério com relevância complexa. Esta metodologia permite a solução de diversos problemas, incluindo a hierarquia de possíveis alternativas (Saaty, 2008; Min, 2010). É um método analítico, que segue estratégias de decomposição de problemas complexos e menos complexos. 
Inicialmente se cria hierarquia (Saaty \& Shih, 2009) que tem o objetivo de descrever o problema. Inicia com o objetivo global na parte superior e decompondo-o em partes, são percebidos como uma estrutura de árvore, tendo como raiz o objetivo global. As comparações paritárias expressam termos linguísticos/verbais que são convertidos em valores numéricos usando a Escala de Saaty para julgamentos comparativos. Os valores desta escala se dão em 9 pontos (Tabela 1). Ela tem objetivo de medir o grau de importância dos elementos levantados.

\section{Tabela 1}

Escala de Saaty

\begin{tabular}{ccc} 
ESCALA & AVALIAÇÃO RECIPROCO \\
\hline Extremamente preferido & 9 & $1 / 9$ \\
Muito forte a extremo & 8 & $1 / 8$ \\
Muito fortemente preferido & 7 & $1 / 7$ \\
Forte a muito forte & 6 & $1 / 6$ \\
Fortemente preferido & 5 & $1 / 5$ \\
Moderado a forte & 4 & $1 / 4$ \\
Moderadamente preferido & 3 & $1 / 3$ \\
Igual a moderado & 2 & $1 / 2$ \\
Igualmente preferido & 1 & 1 \\
\hline
\end{tabular}

Fonte: Saaty (2008).

Após estabelecer os critérios para julgamentos, e utilizar essas informações para calcular os pesos, será possível analisar a consistência das informações.

A consistência se dá quando por meio de um montante de dados brutos de uma base determinada, todos os outros dados podem ser logicamente deduzidos por esta mesma. As vezes os valores podem se mostrar inconsistentes. Diante disso, Saaty desenvolve procedimentos que permitem avaliar a consistência dos julgamentos:

Índice de Consistência (IC) avalia o grau de inconsistência da matriz realizada par a par, por meio da Equação:

$\mathrm{IC}=$

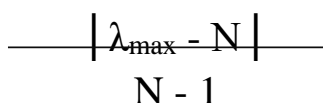

Onde:

$\mathrm{N}$ : é a ordem da matriz.

$\lambda_{\max }$ : o maior autovalor da matriz de julgamentos paritários.

O cálculo do autovalor máximo, utiliza uma tabela auxiliar ( $\left.A^{\prime \prime}\right)$, que é obtida pela multiplicação da 1a coluna da matriz de julgamentos pela prioridade de A, e assim por diante. O cálculo considera 
também um vetor de prioridades auxiliar P", cujo valor é obtido a partir da soma das linhas de A", dividindo os elementos do vetor $\mathrm{P}^{\prime \prime}$, pelos respectivos elementos do vetor de prioridades (Para auxiliar). $\lambda$ máx é calculo que utiliza a soma dos elementos armazenados em $\mathrm{P}$ auxiliar dividida pela ordem da matriz (N).

Razão de Consistência (RC): é calculo que permite avaliar a inconsistência em função dos julgamentos realizados, por meio da Equação:

$$
\mathrm{RC}=\frac{\mathrm{IC}}{\mathrm{IR}}
$$

Onde: IC é o Índice de Consistência e IR é o Índice Randômico (Random Index). O índice Randômico é percebido como o índice de consistência obtido para uma matriz randômica recíproca, apresentando elementos não-negativos, para diversos tamanhos de matriz N.

Na Tabela 2 é possível visualizar a ordem das matrizes e seus respectivos valores IR.

\section{Tabela 2}

Índice de Consistência Randômica

\begin{tabular}{|c|c|c|c|c|c|c|c|c|c|c|}
\hline Dimensão da matriz & 1 & 2 & 3 & 4 & 5 & 6 & 7 & 8 & 9 & 10 \\
\hline $\begin{array}{l}\text { Inconsistência } \\
\text { aleatória }\end{array}$ & 0 & 0 & 0,58 & 0,9 & 1,12 & 1,24 & 1,32 & 1,14 & 1,45 & 1,49 \\
\hline
\end{tabular}

Para a realização de uma matriz aceitável, o pesquisador necessita ter um IR com uma razão de consistência menor que ou igual a $10 \%$.

\section{Discussão dos resultados}

A metodologia AHP foi eleita para a análise dos resultados desta pesquisa. Mediante uma avaliação realizada pela gestora do empreendimento foi possível encontrar a melhor alternativa, diante das dimensões da sustentabilidade, que venha a contribuir para um melhor desempenho da empresa. Por meio da Tabela 3 pode-se perceber os resultados da matriz dos critérios. 
Tabela 3

Tabela com a matriz de Critérios

\begin{tabular}{|c|c|c|c|c|c|c|c|c|c|}
\hline \multicolumn{10}{|c|}{ Matriz de Critérios } \\
\hline & Crl & Cr2 & $\mathrm{Cr} 3$ & $\mathrm{Cr} 4$ & Auto vetor & Vetor normalizado & $\lambda$ máx & IC & $\mathrm{RC}(\%)$ \\
\hline Crl & 1 & 5 & 5 & 7 & 3,64 & $64 \%$ & & & \\
\hline $\mathrm{Cr} 2$ & $1 / 5$ & 1 & 1 & 1 & 0,67 & $12 \%$ & & & \\
\hline $\mathrm{Cr} 3$ & $1 / 5$ & 1 & 1 & 3 & 0,88 & $16 \%$ & 4,07 & 0,02 & $3 \%$ \\
\hline Cr4 & $1 / 7$ & 1 & $1 / 3$ & 1 & 0,47 & $8 \%$ & & & \\
\hline Soma & 1,54 & 8,00 & 7,33 & 12,00 & 5,65 & $100 \%$ & & & \\
\hline
\end{tabular}

Fonte: Elaborado pelos autores.

Ao realizar a comparação par a par, percebeu-se que o $\mathrm{Cr} 1$ obteve $64 \%$, o que indica que a representação mais relevante dos critérios é a qualidade. A qualidade dos pratos é extremamente valorizada pelos consumidores de restaurantes e, assim, acaba por ser fator essencial na fidelização de clientes contribuindo para a sustentabilidade econômica da organização (Franco, Franco \& Naranjo, 2017). Além disso, a análise apontou um índice RC de 3\%, isso representa que há consistência, pois é inferior a 10\%. Na Tabela 4, é possível visualizar a matriz do critério qualidade em relação às alternativas.

\section{Tabela 4}

Tabela resumo do critério Qualidade e alternativas

\begin{tabular}{|c|c|c|c|c|c|c|c|c|c|c|c|c|}
\hline \multicolumn{13}{|c|}{ Qualidade } \\
\hline & $\mathbf{A}$ & B & C & D & $\mathbf{E}$ & F & $\mathbf{G}$ & Auto Vetor & Vetor Normalizado & $\lambda$ máx & IC & $\mathrm{RC}(\%)$ \\
\hline A & 1 & 9 & 5 & 5 & 3 & 3 & 1 & 2,97 & $30 \%$ & & & \\
\hline B & $1 / 9$ & 1 & $1 / 3$ & $1 / 3$ & $1 / 7$ & $1 / 7$ & $1 / 9$ & 0,22 & $2 \%$ & & & \\
\hline C & $1 / 5$ & 3 & 1 & 1 & $1 / 3$ & $1 / 3$ & $1 / 5$ & 0,54 & $5 \%$ & & & \\
\hline D & $1 / 5$ & 3 & 1 & 1 & $1 / 3$ & $1 / 3$ & $1 / 5$ & 0,54 & $5 \%$ & 715 & 0025 & $2 \%$ \\
\hline $\mathbf{E}$ & $1 / 3$ & 7 & 3 & 3 & 1 & 1 & $1 / 3$ & 1,32 & $13 \%$ & 1,13 & 0,023 & $2 \%$ \\
\hline F & $1 / 3$ & 7 & 3 & 3 & 1 & 1 & $1 / 3$ & 1,32 & $13 \%$ & & & \\
\hline $\mathbf{G}$ & 1,00 & 9,00 & 5,00 & 5,00 & 3,00 & 3,00 & 1,00 & 2,97 & $30 \%$ & & & \\
\hline Soma & 3,18 & 39,00 & 18,33 & 18,33 & 8,81 & 8,81 & 3,18 & 9,88 & $100 \%$ & & & \\
\hline
\end{tabular}

Fonte: Elaborado pelos autores.

Através das análises acerca das prioridades gerais, é possível observar que no critério Qualidade (Cr1), a alternativa que se destacou da melhor forma foi a $\mathrm{A}$ e a $\mathrm{G}$ com 30\%, representando o treinamento de pessoal aplicado no ambiente de trabalho do restaurante e a padronização do tempo de preparo das refeições. O treinamento de profissionais, bem como a padronização do tempo para preparar refeições é de extrema importância para o bom andamento de um restaurante e o mesmo influencia tanto no pilar econômico quanto pessoal da sustentabilidade (Dipietro et al., 2007). A implementação de treinamentos e padronização de tempo auxilia nas melhorias do processo e no aumento do bem-estar dos colaboradores contribuindo positivamente para os pilares social e econômico da sustentabilidade. Acerca do julgamento da gestora obteve-se um RC de $2 \%$ isso aponta que há consistência, pois o valor é inferior a 10\%. Na Tabela 5 será apresentado o resumo do critério Custo em relação às alternativas. 


\section{Tabela 5}

Tabela resumo do critério Custo e alternativas

\begin{tabular}{|c|c|c|c|c|c|c|c|c|c|c|c|c|c|c|c|c|}
\hline \multicolumn{17}{|c|}{ Custo } \\
\hline & $\mathbf{A}$ & B & & C & & D & $\mathbf{E}$ & & F & & $\mathbf{G}$ & Auto Vetor & Vetor Normalizado & $\lambda$ máx & IC & $\mathrm{RC}(\%)$ \\
\hline $\mathbf{A}$ & 1 & 3 & & 3 & & 1 & 3 & & 3 & & 1 & 1,87 & $23 \%$ & & & \\
\hline B & $1 / 3$ & 1 & & 1 & & $1 / 3$ & 1 & & 1 & & $1 / 3$ & 0,62 & $8 \%$ & & & \\
\hline C & $1 / 3$ & 1 & & 1 & & $1 / 3$ & 1 & & 1 & & $1 / 3$ & 0,62 & $8 \%$ & & & \\
\hline D & 1 & 3 & & 3 & & 1 & 3 & & 3 & & 1 & 1,87 & $23 \%$ & 700 & 0 & $0 \%$ \\
\hline $\mathbf{E}$ & $1 / 3$ & 1 & & 1 & & $1 / 3$ & 1 & & 1 & & $1 / 3$ & 0,62 & $8 \%$ & $\gamma, 00$ & 0 & 070 \\
\hline $\mathbf{F}$ & $1 / 3$ & 1 & & 1 & & $1 / 3$ & 1 & & 1 & & $1 / 3$ & 0,62 & $8 \%$ & & & \\
\hline $\mathbf{G}$ & 1 & 3 & & 3 & & 1 & 3 & & 3 & & 1 & 1,87 & $23 \%$ & & & \\
\hline Soma & 4,33 & & 13 & & 13 & 4,33 & & 13 & & 13 & 4,33 & 8,12 & $100 \%$ & & & \\
\hline
\end{tabular}

Fonte: Elaborado pelos autores.

No que tange ao $\mathrm{Cr} 2$ (Custo) as alternativas que obtiveram maior destaque foram a $\mathrm{A}$, a $\mathrm{D}$ e a $\mathrm{G}$ com $23 \%$, a primeira representa o treinamento do pessoal, a segunda demonstra o gerenciamento de matéria prima e o terceiro aponta a padronização do tempo. Tanto o treinamento dos colaboradores, quanto o gerenciamento adequado da matéria-prima e a padronização do tempo são de extrema importância para a organização, o gerenciamento, por exemplo, evita desperdícios monetários e contaminação dos insumos por micro-organismos causadores de doenças aos consumidores do produto (Mitchell \& Harris, 2005). Essas três alternativas contribuem diretamente os três pontos do tripé da sustentabilidade, melhorando o bem-estar dos colaboradores, reduzindo desperdícios e diminuindo custos de produção. Acerca da consistência da matriz diz-se que a mesma é perfeitamente consistente pois apresenta RC de $0 \%$. A partir da Tabela 6 é possível observar o resumo do critério Tempo em relação às alternativas.

\section{Tabela 6}

Tabela resumo do critério Tempo e alternativas

\begin{tabular}{|c|c|c|c|c|c|c|c|c|c|c|c|c|}
\hline \multicolumn{13}{|c|}{ Tempo } \\
\hline & A & B & $\mathbf{C}$ & D & $\mathbf{E}$ & $\mathbf{F}$ & $\mathbf{G}$ & Auto Vetor & Vetor Normalizado & $\lambda$ máx & IC & $\mathrm{RC}(\%)$ \\
\hline $\mathbf{A}$ & 1 & 3 & 7 & 5 & 5 & 3 & 1 & 2,86 & $30 \%$ & & & \\
\hline B & $1 / 3$ & 1 & 3 & 1 & 1 & $1 / 3$ & $1 / 5$ & 0,68 & $7 \%$ & & & \\
\hline C & $1 / 7$ & $1 / 3$ & 1 & $1 / 3$ & $1 / 3$ & $1 / 5$ & $1 / 7$ & 0,28 & $3 \%$ & & & \\
\hline D & $1 / 5$ & 1 & 3 & 1 & 1 & $1 / 3$ & $1 / 5$ & 0,63 & $7 \%$ & & & $2 \%$ \\
\hline $\mathbf{E}$ & $1 / 5$ & 1 & 3 & 1 & 1 & $1 / 3$ & $1 / 5$ & 0,63 & $7 \%$ & 7,19 & 0,03 & $2 \%$ \\
\hline $\mathbf{F}$ & $1 / 3$ & 3 & 5 & 3 & 3 & 1 & $1 / 3$ & 1,47 & $15 \%$ & & & \\
\hline G & 1 & 5 & 7 & 5 & 5 & 3 & 1 & 3,08 & $32 \%$ & & & \\
\hline Soma & 3,21 & 14,33 & 29,00 & 16,33 & 16,33 & 8,20 & 3,08 & 9,64 & $100 \%$ & & & \\
\hline
\end{tabular}

Fonte: Elaborado pelos autores.

A partir do julgamento da gestora para o CR3 (Tempo), a alternativa G obteve maior destaque com $32 \%$ do total, a qual representa a padronização do tempo. A padronização do tempo garante que a empresa reduza seus custos e, consequentemente, diminua os desperdícios no processo contribuindo diretamente com os pilares econômico e ambiental da sustentabilidade (White, 2002). Além disso, percebeu-se, também que o RC resultou em $2 \%$ e isso aponta que o julgamento é consistente, pois é 
inferior ao valor de 10\%. Na Tabela 7 será demonstrado o resumo do Critério Recursos em relação às alternativas.

Tabela 7

Tabela resumo do critério Recursos e alternativas

\begin{tabular}{|c|c|c|c|c|c|c|c|c|c|c|c|c|}
\hline \multicolumn{13}{|c|}{ Recursos } \\
\hline & A & B & C & D & E & F & G & Auto Vetor & Vetor Normalizado & $\lambda$ máx & IC & $\mathrm{RC}(\%)$ \\
\hline A & 1 & 7 & 7 & 3 & 3 & 1 & 3 & 2,79 & $29 \%$ & & & \\
\hline B & $1 / 7$ & 1 & 1 & $1 / 5$ & $1 / 5$ & $1 / 7$ & $1 / 5$ & 0,29 & $3 \%$ & & & \\
\hline C & $1 / 7$ & 1 & 1 & $1 / 5$ & $1 / 5$ & $1 / 7$ & $1 / 5$ & 0,29 & $3 \%$ & & & \\
\hline D & $1 / 3$ & 5 & 5 & 1 & 1 & $1 / 3$ & 1 & 1,16 & $12 \%$ & 71 & 007 & $\%$ \\
\hline E & $1 / 3$ & 5 & 5 & 1 & 1 & $1 / 3$ & 1 & 1,16 & $12 \%$ & $\gamma, 15$ & 0,02 & $2 \%$ \\
\hline F & 1 & 7 & 7 & 3 & 3 & 1 & 3 & 2,79 & $29 \%$ & & & \\
\hline G & $1 / 3$ & 5 & 5 & 1 & 1 & $1 / 3$ & 1 & 1,16 & $12 \%$ & & & \\
\hline Soma & 3,29 & 31,00 & 31,00 & 9,40 & 9,40 & 3,29 & 9,40 & 9,63 & $100 \%$ & & & \\
\hline
\end{tabular}

Fonte: Elaborado pelos autores.

Ao analisar os julgamentos para o CR4 (Recursos), as alternativas mais relevantes foram A e $\mathrm{F}$ com 29\%, o que representa o treinamento de pessoal e estoque. Treinar pessoal e gerenciar corretamente estoques contribui diretamente para que os alimentos sejam bem conservados e preparados de forma segura e livre de microrganismos (Gonzalez-Mancebo et al., 2019). Ademais, percebe-se que o RC resultou em $2 \%$ o que demonstra que há consistência nos julgamentos, pois este índice é menor que $10 \%$ o valor máximo de tolerância de erros.

A partir das avaliações efetuadas acerca dos critérios versus alternativas, constata-se que RC da matriz de comparação necessita ser inferior a 10\%. Diante disso, o $\mathrm{Cr} 1$ obteve $2 \%$, seguido do $\mathrm{Cr} 2 \mathrm{com}$ 0 \%, CR3 2\% e CR4 com 2\%. Então, esse julgamento é consistente. Na Tabela 8 serão apresentados os resultados gerais para critérios e alternativas com maior importância.

\section{Tabela 8}

Resultados gerais para critérios e alternativas

\begin{tabular}{cccccr}
\hline \multicolumn{7}{c}{ Critérios vs. Alternativas } \\
\hline Alternativas & Qualidade & Custo & Tempo & Recursos & \% Total \\
Vetor dos criterios & $\mathbf{6 4} \%$ & $12 \%$ & $16 \%$ & $8 \%$ & \\
A & $30 \%$ & $23 \%$ & $30 \%$ & $29 \%$ & $\mathbf{2 9} \%$ \\
B & $2 \%$ & $8 \%$ & $7 \%$ & $3 \%$ & $4 \%$ \\
C & $5 \%$ & $8 \%$ & $3 \%$ & $3 \%$ & $5 \%$ \\
D & $5 \%$ & $23 \%$ & $7 \%$ & $12 \%$ & $8 \%$ \\
E & $13 \%$ & $8 \%$ & $7 \%$ & $12 \%$ & $12 \%$ \\
F & $13 \%$ & $8 \%$ & $15 \%$ & $29 \%$ & $14 \%$ \\
G & $30 \%$ & $23 \%$ & $32 \%$ & $12 \%$ & $28 \%$ \\
\multicolumn{6}{c}{ coluna } \\
\hline
\end{tabular}

Fonte: Elaborado pelos autores. 
Ao observar a Tabela 8, percebe-se análise dos dados, onde percebe-se que o critério que mais se destacou foi a Qualidade (64\%) e a alternativa mais relevante foi a A, com uma ponderação de $29 \%$. Essa alternativa refere-se ao treinamento de colaboradores e, por meio desse resultado pode-se perceber a importância que essa prática tem em uma empresa do ramo alimentício. A qualidade está intimamente ligada ao treinamento de pessoal, pois colaboradores bem treinados possuem maior capacidade de produzir bens padronizados e com um maior valor de venda (Bilanakos et al., 2018). Diante disso, é imprescindível que a organização conheça os seus processos a fim de melhorar a qualidade de seus processos e produtos de modo que a mesma possa transformar seus processos a partir de conceitos da produção enxuta.

\subsection{Análise de sensibilidade}

Diante as análises efetuadas através do método AHP, verificou-se a alternativa a ser priorizada pelo gestor do restaurante. Entretanto, com o intuito de verificar a veracidade e a robustez da avaliação, bem como, as mudanças que poderiam ocorrer com variações recorrentes nos pesos relativos e ainda no ranking final das alternativas, elaborou-se uma análise de sensibilidade. Autores denotam que utilizando esse modelo de análise, é possível verificar ambientes de decisão alternativos, ainda, pontos de vistas diferentes no que tange a importância dos critérios avaliados (Chang et al., 2007; Mangla; Kumar \& Barua, 2015).

Para cada critério (Qualidade, Tempo; Custo e Recursos), elaborou-se simulações, visando verificar o comportamento da decisão final no que tange a esses critérios. Na Figura 3, é possível visualizar o comportamento do ranking das alternativas com os pesos em mudanças para cada critério. 
Figura 3

Simulação de mudança dos pesos do Critério Qualidade

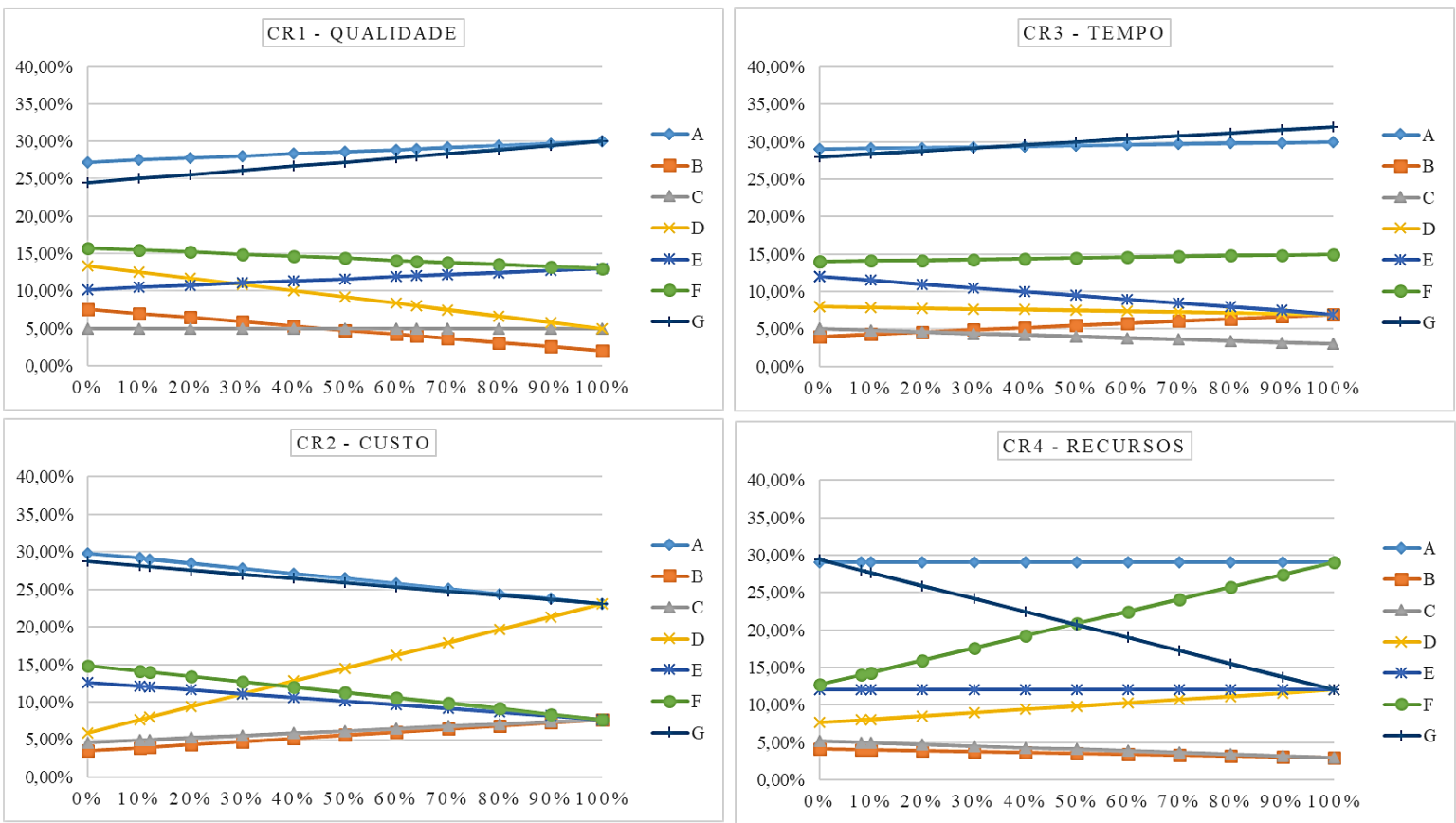

Fonte: Elaborado pelos autores.

Observando a Figura 3, torna-se possível visualizar o comportamento das alternativas com a mudança de pesos relativos.

Denota-se que os pesos dos critérios foram individualmente modificados, obtendo uma simulação que variou de 0\% a 100\%. Para Chang et al. (2007), a análise de sensibilidade torna-se importante, pois a mudança dos pesos dos critérios, requerem vários níveis diferenciados de gerenciamento.

\section{Considerações finais}

O objetivo do presente estudo foi avaliar as alternativas de melhoria na qualidade no processo com ênfase nas práticas sustentáveis em um restaurante situado na região noroeste do Rio Grande do Sul.

Diante a aplicação do modelo multicritério AHP, pode-se priorizar as alternativas que visavam a melhoria da qualidade nos processos do restaurante estudado, alinhando as mesmas, as práticas sustentáveis aspecto esse que tem demonstrado de suma importância atualmente.

Percebe-se que o método tem sido utilizado em diversas pesquisas voltadas a temática da sustentabilidade, demonstrando sua eficácia em auxiliar na tomada de decisão (Wang et al., 2019; Bhandari, Singh \& Garg, 2019; Aguilar-Rivera, 2019; Wankhede, Digalwar \& Wankhede, 2019). A presente pesquisa, vem contribuir com o desenvolvimento de estudos que versam a respeito de práticas em empresas de um ramo de suma importância para a economia, gerando com isso mudanças no 
comportamento dos colaboradores e gestores, frente a sustentabilidade, aumentando o aporte teórico e prático para futuros pesquisadores da área.

Com análise prévia do restaurante, os pesquisadores puderam verificar pontos vitais que demandavam melhoria. Utilizando o embasamento teórico, foi possível propor alternativas que viessem auxiliar o restaurante analisado a melhorar, tanto no aspecto da qualidade quanto nas questões relacionadas à sustentabilidade.

Utilizando a expertise do gestor da organização, pode-se denotar que a alternativa prioritária para que o restaurante implementasse melhorias significativas está relacionada à treinamento de pessoal. A adoção de novas práticas resulta em redução de desperdícios.

Elucida-se que, com a análise de sensibilidade efetuada, pode-se verificar o comportamento das variáveis, indicando nas simulações, cenários alternativos que poderiam demonstrar a sensibilidade existente na tomada de decisão.

Como sugestão para estudos futuros, cita-se a utilização de modelos de simulação para verificar o impacto gerado com a aplicação da alternativa priorizada e ainda um estudo que verse a respeito da destinação dos resíduos gerados pela organização. Pois estudos recentes têm demonstrado que muitos resíduos advindos até mesmo de restaurantes podem ser potenciais alternativas, como no caso do óleo de cozinha usado, que pode ser matéria prima para fabricação de biodiesel (Bechet et al., 2010; Demirbas, 2009; Zhang \& Kong, 2010; Mendes, 2015; Aboelazayem, Gadalla \& Saha, 2019; Aghel et al., 2019; Ahmad et al., 2019; Nabi et al., 2019; Velmurugan et al., 2019; Tan et al., 2019; Mohadesi et al., 2019; Dimitriou, Tsujimura \& Suzuki, 2019).

Dessa forma, entende-se que o objetivo delineado fora alcançado, demonstrando que os restaurantes além de pensar na qualidade, devem pensar também em ser sustentáveis, alinhando suas práticas a esse conceito.

\section{Referências}

Aboelazayem, O., Gadalla, M., \& Saha, B. (2019). Derivatisation-free characterisation and supercritical conversion of free fatty acids into biodiesel from high acid value waste cooking oil. Renewable Energy, 143, 77-90. https://doi.org/10.1016/j.renene.2019.04.106

Aghazadeh, S. M. (2005). Layout strategies for retail operations: A case study. Management Research News, 28(10), 31-46. https://doi.org/10.1108/01409170510785002

Aghel, B., Mohadesi, M., Ansari, A., \& Maleki, M. (2019). Pilot-scale production of biodiesel from waste cooking oil using kettle limescale as a heterogeneous catalyst. Renewable Energy, 142, 207214. https://doi.org/10.1016/j.renene.2019.04.100

Aguiar, M. A. F. (2002). Psicologia aplicada à administração: globalização, pensamento complexo, teoria crítica e a questão ética nas organizações. 3.ed. São Paulo: Excellus Editora. 
Aguilar-Rivera, N. (2019). A framework for the analysis of socioeconomic and geographic sugarcane agro industry sustainability. Socio-Economic Planning Sciences, 66, 149-160. https://doi.org/10.1016/j.seps.2018.07.006

Ahmad, N., Javed, F., Awan, J. A., Ali, S., Fazal, T., Hafeez, A., ... \& Rehman, F. (2019). Biodiesel production intensification through microbubble mediated esterification. Fuel, 253, 25-31. https://doi.org/10.1016/j.fuel.2019.04.173

Alegre, I., \& Berbegal-Mirabent, J. (2018). Gourmet Products from Food Waste: Rethinking Food Management and Social Justice. Handbook of Engaged Sustainability, 683-702. https://doi.org/10.1007/978-3-319-71312-0_22

Appolinário, F. (2013). Dicionário de metodologia científica: um guia para a produção do conhecimento científico. 2.ed. São Paulo: Atlas.

Batista, A. P., Gouveia, L., \& Marques, P. A. (2018). Fermentative hydrogen production from microalgal biomass by a single strain of bacterium Enterobacter aerogenes-Effect of operational conditions and fermentation kinetics. Renewable energy, 119, 203-209. https://doi.org/10.1016/j.renene.2017.12.017

Bechet, Q., Shilton, A., Fringer, O. B., Munoz, R., \& Guieysse, B. (2010). Mechanistic modeling of broth temperature in outdoor photobioreactors. Environmental science \& technology, 44(6), 21972203. https://doi.org/10.1021/es903214u

Bhandari, D., Singh, R. K., \& Garg, S. K. (2019). Prioritisation and evaluation of barriers intensity for implementation of cleaner technologies: Framework for sustainable production. Resources, Conservation and Recycling, 146, 156-167. https://doi.org/10.1016/j.resconrec.2019.02.038

Bharucha, J. (2018). Tackling the challenges of reducing and managing food waste in Mumbai restaurants. British Food Journal, 120(3), 639-649. https://doi.org/10.1108/BFJ-06-2017-0324

Bilanakos, C., Heywood, J. S., Sessions, J., \& Theodoropoulos, N. (2018). Does demand for product quality increase worker training?. Journal of Economic Behavior \& Organization, 155, 159-177. https://doi.org/10.1016/j.jebo.2018.08.018

Brandalise, L. T., Bertolini, G. R., Rojo, C. A., Lezana, Á. G., \& Possamai, O. (2009). A percepção e o comportamento ambiental dos universitários em relação ao grau de educação ambiental. Revista Gestão \& Produção, 16(2), 286-300. https://doi.org/10.1590/S0104$530 \times 2009000200010$

Cauchick Miguel, P.A. (org). (2012). Metodologia de Pesquisa em Engenharia de Produção e Gestão e Operações. Elsevier.

Collis, J., \& Hussey, R. (2005). Pesquisa em administração: um guia prático para alunos de graduação e pós-graduação. Bookman.

Chang, C. W., Wu, C. R., Lin, C. T., \& Chen, H. C. (2007). An application of AHP and sensitivity analysis for selecting the best slicing machine. Computers \& Industrial Engineering, 52(2), 296-307. https://doi.org/10.1016/j.cie.2006.11.006

Demo, P. (2013). Introdução à metodologia da ciência.2. ed. 21. reimpr. São Paulo: Atlas. 
Demirbas, M. F. (2009). Biorefineries for biofuel upgrading: a critical review. Applied Energy, 86, S151S161. https://doi.org/10.1016/j.apenergy.2009.04.043

Dimitriou, P., Tsujimura, T., \& Suzuki, Y. (2019). Adopting biodiesel as an indirect way to reduce the NOx emission of a hydrogen fumigated dual-fuel engine. Fuel, 244, 324-334. https://doi.org/10.1016/j.fuel.2019.02.010

DiPietro, R. B., Murphy, K. S., Rivera, M., \& Muller, C. C. (2007). Multi-unit management key success factors in the casual dining restaurant industry: A case study. International journal of contemporary hospitality management, 19(7), 524-536. https://doi.org/10.1108/09596110710818275

Ergün, N., \& Bülbül, K. G. (2019). An assessment of factors affecting airport security services: an AHP approach and case in Turkey. Security Journal, 32(1), 20-44. https://doi.org/10.1057/s41284018-0143-4

Florén, H., Frishammar, J., Löf, A., \& Ericsson, M. (2019). Raw materials management in iron and steelmaking firms. Mineral Economics, 32(1), 39-47. https://doi.org/10.1007/s13563-0180158-7

Franco, M. C., Franco, W. C., \& Naranjo, M. T. (2017). Análisis de satisfacción: La gastronomía de Samborondón-Ecuador. Estudios y perspectivas en turismo, 26(3), 731-745.

Garcia, S., Cordeiro, A., de Alencar Nääs, I., \& Neto, P. L. D. O. C. (2019). The sustainability awareness of Brazilian consumers of cotton clothing. Journal of Cleaner Production, 215, 1490-1502. https://doi.org/10.1016/j.jclepro.2019.01.069

Gigli, S., Landi, D., \& Germani, M. (2019). Cost-benefit analysis of a circular economy project: a study on a recycling system for end-of-life tyres. Journal of Cleaner Production, 229, 680-694. https://doi.org/10.1016/j.jclepro.2019.03.223

Gil, A. C. (2010). Como elaborar projetos de pesquisa. São Paulo: Atlas. 184p.

Gonzalez-Mancebo, E., Gandolfo-Cano, M. M., Trujillo-Trujillo, M. J., Mohedano-Vicente, E., Calso, A., Juarez, R., ... \& Pajuelo, F. (2019). Analysis of the effectiveness of training school personnel in the management of food allergy and anaphylaxis. Allergologia et immunopathologia, 47(1), 60-63. https://doi.org/10.1016/j.aller.2018.05.005

Hashem, M., Asseri, T. Y., Alamri, S. A., \& Alrumman, S. A. (2019). Feasibility and Sustainability of Bioethanol Production from Starchy restaurants' Bio-wastes by New Yeast Strains. Waste and Biomass Valorization, 10(6), 1617-1626. https://doi.org/10.1007/s12649-017-0184-7

Ho, W., Dey, P. K., \& Lockström, M. (2011). Strategic sourcing: a combined QFD and AHP approach in manufacturing. Supply Chain Management: An International Journal, 16(6), 446-461. https://doi.org/10.1108/13598541111171093

Kim, H. S., \& Shim, J. H. (2019). The effects of quality factors on customer satisfaction, trust and behavioral intention in chicken restaurants. Journal of Industrial Distribution \& Business Vol, 10(4), 43-56. https://doi.org/10.13106/ijidb.2019.vol10.no4.43 
Koo, D. W. (2018). The Impact of Risk Perceptions of Food Ingredients on the Restaurant Industry: Focused on the Moderating Role of Corporate Social Responsibility. Sustainability, 10(9), 3132. https://doi.org/10.3390/su10093132

Lee, S. H. (2010). Using fuzzy AHP to develop intellectual capital evaluation model for assessing their performance contribution in a university. Expert systems with applications, 37(7), 4941-4947. https://doi.org/10.1016/j.eswa.2009.12.020

Madruga, L.R.R.G. (2009). Comportamento coletivo e interações sociais no comitê de gerenciamento da bacia hidrográfica do rio Santa Maria: aprendizagem social e emergência do empreendedorismo socioambiental. Tese de Doutorado Universidade Federal do Rio Grande do Sul. Porto Alegre.

Lakatos, E. M., \& Marconi, M. D. A. (2010). Fundamentos de metodologia científica. 5. reimp. São Paulo: Atlas, 310.

Mangla, S. K., Kumar, P., \& Barua, M. K. (2015). Risk analysis in green supply chain using fuzzy AHP approach: A case study. Resources, Conservation and Recycling, 104, 375-390. https://doi.org/10.1016/j.resconrec.2015.01.001

Martins, R. A., Mello, J. B. P., \& Turrioni, C. H. (2014). Guia para elaboração de monografia e TCC em engenharia de produção. Editora Atlas SA.

Mastrapa Gutiérrez, B., \& Sánchez Batista, A. (2017). Sistema integrado de costos de calidad y medio ambiente para la gestión y la sostenibilidad empresarial. Retos de la Dirección, 11(1), 21-37.

Mendes, P.A.S. (2015). Sustentabilidade na produção e uso do biodiesel. Curitiba. Appris.

Michalec, A., Fodor, M., Hayes, E., \& Longhurst, J. (2018). Co-designing food waste services in the catering sector. British Food Journal, 120(12), 2762-2777. https://doi.org/10.1108/BFJ-042018-0226

Min, H. (2010). Evaluating the comparative service quality of supermarkets using the analytic hierarchy process. Journal of Services Marketing, 24(4), 283-293.

https://doi.org/10.1108/08876041011052999

Mitchell, V. W., \& Harris, G. (2005). The importance of consumers' perceived risk in retail strategy. European Journal of marketing, 39(7/8), 821-837.

https://doi.org/10.1108/03090560510601789

Mohadesi, M., Aghel, B., Maleki, M., \& Ansari, A. (2019). Production of biodiesel from waste cooking oil using a homogeneous catalyst: Study of semi-industrial pilot of microreactor. Renewable Energy, 136, 677-682. https://doi.org/10.1016/j.renene.2019.01.039

Nabi, M. N., Rasul, M. G., Anwar, M., \& Mullins, B. J. (2019). Energy, exergy, performance, emission and combustion characteristics of diesel engine using new series of non-edible biodiesels. Renewable Energy. https://doi.org/10.1016/j.renene.2019.03.066

Nakuja, T., \& Kerr, W. A. (2018). Food security though public stockholdings and trade distortions. British Food Journal, 120(5), 1089-1105. https://doi.org/10.1108/BFJ-07-20170391 
ONU. (2015) Transformando o nosso mundo: a agenda 2030 para o desenvolvimento sustentável. Disponível em: https://nacoesunidas.org/pos2015/agenda2030/. Acesso em 14 de maio de 2019.

Pakdil, F., Toktaş, P., \& Leonard, K. M. (2018). Validation of qualitative aspects of the Lean Assessment Tool (LAT). Journal of Manufacturing Technology Management, 29(7), 1094-1114. https://doi.org/10.1108/JMTM-12-2017-0268

Peterková, J., \& Franek, J. (2018). Specifics of management of enterprise innovation activities in the Czech republic-the decision-making mechanism. https://doi.org/10.1515/mspe-2018-0022

Porter, M. E. (1996) Condensado de What is Strategy? Harvard Business Review.

Raab, C., Baloglu, S., \& Chen, Y. S. (2018). Restaurant Managers' Adoption of Sustainable Practices: An Application of Institutional Theory and Theory of Planned Behavior. Journal of foodservice business research, 21(2), 154-171. https://doi.org/10.1080/15378020.2017.1364591

Raval, S. J., Kant, R., \& Shankar, R. (2018). Revealing research trends and themes in Lean Six Sigma: from 2000 to 2016. International Journal of Lean Six Sigma, 9(3), 399-443. https://doi.org/10.1108/IJLSS-03-2017-0021

Ruiz, J. Á. (2011). Metodologia Científica: guia para eficiência nos estudos. -5. reimpr. São Paulo: Atlas.

Saaty, T. L. (2008). Decision making with the analytic hierarchy process. International journal of services sciences, 1(1), 83-98. https://doi.org/10.1504/IJSSCI.2008.017590

Saaty, T. L., \& Shih, H. S. (2009). Structures in decision making: On the subjective geometry of hierarchies and networks. European Journal of Operational Research, 199(3), 867-872. https://doi.org/10.1016/j.ejor.2009.01.064

Salzberg, A. C., Gough, M. Z., \& Suen, I. S. (2019). Sustainable innovation behavior in restaurants. Journal of Foodservice Business Research, 1-24. https://doi.org/10.1080/15378020.2019.1592655

Shen, Jianming et al. An improved emergy evaluation of the environmental sustainability of China's steel production from 2005 to 2015. Ecological Indicators, v. 103, p. 55-69, 2019. https://doi.org/10.1016/j.ecolind.2019.03.051

Shunmugasundaram, M., Maneiah, D. (2018). Wastage minimization and manufacturing cost reduction in raw edge cogged belts by lean manufacturing method. International Journal of Mechanical Engineering and Technology, 9 (7), pp. 678-686.

Tan, Y. H., Abdullah, M. O., Kansedo, J., Mubarak, N. M., San Chan, Y., \& Nolasco-Hipolito, C. (2019). Biodiesel production from used cooking oil using green solid cabtalyst derived from calcined fusion waste chicken and fish bones. Renewable Energy, 139, 696-706. https://doi.org/10.1016/j.renene.2019.02.110

Telles, F. S., Pérez, D. M., López-Espinoza, A., \& Teyes, E. S. (2013). Comportamiento y organización. Implementación del sistema de gestión de la calidad 5S'S. Diversitas, 9(2), 361-371. https://doi.org/10.15332/s1794-9998.2013.0002.09 
Teng, Y. M., \& Wu, K. S. (2019). Sustainability Development in Hospitality: The Effect of Perceived Value on Customers' Green Restaurant Behavioral Intention. Sustainability, 11(7), 1987. https://doi.org/10.3390/su11071987

Tommasetti, A., Singer, P., Troisi, O., \& Maione, G. (2018). Extended Theory of Planned Behavior (ETPB): Investigating Customers' Perception of Restaurants' Sustainability by Testing a Structural Equation Model. Sustainability, 10(7), 2580. https://doi.org/10.3390/su10072580

Trel'ová, S.; Olšavský, F. (2017). Investment in education and training of employees of the company/Legal and financial aspects. Proceedings of the 29th International Business Information Management Association (IBIMA).

Trudel, R. (2019). Sustainable consumer behavior. Consumer Psychology Review, 2(1), 85-96. https://doi.org/10.1002/arcp.1045

Ulutaş, A., Topal, A., \& Bakhat, R. (2019). An Application of Fuzzy Integrated Model in Green Supplier Selection. Mathematical Problems in Engineering, 2019. https://doi.org/10.1155/2019/4256359

Velmurugan, R., Mayakrishnan, J., Induja, S., Raja, S., Nandagopal, S., \& Sathyamurthy, R. (2019). Comprehensive Study on the Effect of $\mathrm{CuO}$ Nano Fluids Prepared Using One-Step Chemical Synthesis Method on the Behavior of Waste Cooking Oil Biodiesel in Compression Ignition Engine. Journal of Thermal Science and Engineering Applications, 11(4), 041003. https://doi.org/10.1115/1.4041878

Vinck, K., Scheelen, L., \& Du Bois, E. (2019). Design opportunities for organic waste recycling in urban restaurants. Waste Management \& Research, 37(1_suppl), 40-50.

Wang, Z., Wang, Y., Xu, G., \& Ren, J. (2019). Sustainable desalination process selection: Decision support framework under hybrid information. Desalination, 465, 44-57. https://doi.org/10.1177/0734242X18817714

Wang, Z., Wang, Y., Xu, G., \& Ren, J. (2019). Sustainable desalination process selection: Decision support framework under hybrid information. Desalination, 465, 44-57. https://doi.org/10.1016/j.desal.2019.04.022

Wankhede, S., Digalwar, A. K., \& Wankhede, S. P. (2019, May). Analysis of sustainable machining process: A multicriteria decision making approach. In AIP Conference Proceedings (Vol. 2104, No. 1, p. 030026). AIP Publishing. https://doi.org/10.1063/1.5100453

White, R. (2002). The perils of processed herbs and spices: The encapsulation of herbs and spices as an effective method to eliminate E. coli, Salmonella and other pathogens. British Food Journal, 104(9), 724-729. https://doi.org/10.1108/00070700210443101

Zhang, L., \& Kong, S. C. (2010). Vaporization modeling of petroleum-biofuel drops using a hybrid multi-component approach. Combustion and Flame, 157(11), 2165-2174. https://doi.org/10.1016/j.combustflame.2010.05.011

Zhang, T., Zhao, F., Zhang, J., Mendis, G., Ru, Y., \& Sutherladn, J. W. (2019). An Approximation of the Customer Waiting Time for Online Restaurants Owning Delivery System. Journal of Systems Science and Complexity, 32(3), 907-931. https://doi.org/10.1007/s11424-018-7316-4

Zhu, X., \& Yu, L. (2019). The Impact of Warranty Efficiency of Remanufactured Products on Production Decisions and Green Growth Performance in Closed-Loop Supply Chain: Perspective of Consumer Behavior. Sustainability, 11(5), 1420. https://doi.org/10.3390/su11051420 